$98 \%$ of practitioners in this survey had undergone training in the measurement of blood pressure.

1 GDPs do not want to be involved in screening for hypertension.

1 Most practitioners are keen on professional education about hypertension.

\title{
Blood pressure measuring equipment in the dental surgery: use or ornament?
}

\author{
M. Greenwood ${ }^{1}$ and R. J. Lowry ${ }^{2}$
}

Objective To survey the attitudes, knowledge and practice of general dental practitioners (GDPs) with regard to hypertension in dental patients and to assess opinion with regard to the concept of screening.

Setting General dental practice.

Subjects and Methods GDPs via a postal survey. To achieve an acceptable level of accuracy, at least 196 responses were required. Results Out of 300 questionnaires, 207 were returned (69\% response rate). Most practitioners (98\%) had received training in the measurement of blood pressure. Only $4.8 \%$ measured blood pressure routinely and this figure rose to $9.2 \%$ in patients with a known history of hypertension. Only $27.1 \%$ felt that the involvement of dentists in screening for hypertension was a good idea but $85.3 \%$ thought that education of practitioners about hypertension would be valuable.

Conclusion Most GDPs thought that education of practitioners about hypertension was a good idea. They did not, however, want to be involved with screening of patients for hypertension.

The aim of this study was to survey the attitudes and practices of general dental practitioners (GDPs) in the Northern Region of England with regard to various aspects of hypertension, including the concept of screening patients for hypertension by dentists. In some parts of America, dentists are encouraged to screen all their adult patients for hypertension. ${ }^{1}$ Hypertension is an important but usually asymptomatic clinical condition. Dentists are one group of healthcare professionals who see patients with hypertension that may be undetected, untreated or inadequately treated. Hypertension has been shown to be the most common systemic disease found in elderly dental patients ${ }^{2}$ and may be exacerbated by both the anticipation of dental treatment as well as the treatment itself. ${ }^{3}$

${ }^{1 *}$ Lecturer in Oral and Maxillofacial Surgery ${ }^{2}$ Consultant in Public Health Medicine The Dental School, University of Newcastle upon Tyne, Framlington Place Newcastle upon Tyne NE2 4BW.

*Correspondence to: Mark Greenwood, Lecturer in Oral and Maxillofacial Surgery The Dental School, University of Newcastle upon Tyne, Framlington Place Newcastle upon Tyne NE2 4BW

\section{Refereed paper}

Received 05.11.01; Accepted 30.05.02

(๑) British Dental Journal 2002; 193: 273-275

\section{METHODS}

In this prospective study, questionnaires were sent out to a random sample of GDPs contracted to provide NHS dentistry with Health Authorities in the North East of England. Practitioners on a Specialist List were identified from the Dentist's Register and were excluded from the study with the aim of targeting practitioners carrying out 'general' dental treatment in primary care. Non-responders to the questionnaire were mailed a reminder. It was calculated that with a sample of 196 subjects the probability of the responses for this sample being within $7 \%$ of the total national population of GDPs would be $95 \%$. Three hundred questionnaires (Appendix I) were sent out with the aim of achieving at least 196 responses. In a pilot study, the questionnaire took no longer than 10 minutes to complete.

\section{RESULTS}

Of 300 questionnaires mailed out, 207 were returned completed (a 69\% response rate). There was a wide range of years since qualification of the practitioners (3-31 years). About half $(46.9 \%)$ of the practitioners had either past or current experience of working in hospital practice (Table 1).

\begin{tabular}{lc} 
Table 1 Responses to Hypertension Questionnaire & \\
\hline & \\
Question & Response \\
\hline 1. Years in practice & $3-31$ years \\
2. Hospital position (present/past) & $46.9 \%$ \\
3. Teaching about BP measurement & $62.8 \%$ \\
$\quad \quad$ Dental School & $20.8 \%$ \\
$\quad$ Postgraduate & $14.5 \%$ \\
$\quad$ Both & $1.9 \%$ \\
$\quad$ Never & $85 \%$ \\
4. Specific questioning about hypertension & $82.1 \%$ \\
5. Possession of equipment to measure BP & $4.8 \%$ \\
6. Routine measurement of BP & $9.2 \%$ \\
7. Measurement in hypertensives & $41.1 \%$ \\
8. Practitioners using IV sedation & $89.4 \%$ \\
9. Practitioners checking BP before sedation & $27.1 \%$ \\
10. Practitioners feeling that education about hypertension a good idea. & $85.3 \%$ \\
11. Practitioners feeling that screening a good idea &
\end{tabular}

*For example, if 50\% of all GDPs measure blood pressure and repeated samples of 196 practitioners are taken, in $95 \%$ of samples of this size the proportion detected will be between $43 \%$ and $57 \%$. 
Nearly all practitioners (98.1\%) had received training in the measurement of blood pressure and $62.8 \%$ had received this at dental school. Most practitioners enquired about raised blood pressure $(85 \%)$ whilst taking a medical history and only slightly less (82.1\%) possessed equipment capable of measuring it. Despite this however, only $4.8 \%$ of practitioners reported measuring blood pressure routinely and this figure rose to $9.2 \%$ when assessing patients with a known history of hypertension. Intravenous sedation was used by $41.1 \%$ of practitioners in this survey and of those practitioners using sedation, 89.4\% reported carrying out a pre-sedation blood pressure check.

Most practitioners (95.7\%) gave a reasonable figure for what might be accepted as a 'normal' blood pressure and of these, $70.7 \%$ correctly stated that a range of values around $120 \mathrm{~mm}$ of mercury over $80 \mathrm{~mm}$ of mercury are acceptable. Diuretics and beta blockers were named by $82.1 \%$ and $77.8 \%$ of practitioners respectively and were by far the best known drugs used in the treatment of hypertension.

Only 27.1\% felt that the involvement of dentists in screening for hypertension was a good idea but $85.3 \%$ thought that education of practitioners about hypertension would be valuable.

\section{DISCUSSION}

Except when sedating patients (where blood pressure measurement is mandatory ${ }^{4}$ ) most of the dental practitioners surveyed did not make a habit of taking a blood pressure reading, even though many possessed the necessary equipment and expertise and knew what 'normal' is. Many stated that a lack of a fee on the NHS was the cause of this reluctance, but there may be more deep seated reasons as well. Other possible reasons for not taking blood pressure measurements include not being sure what to do if an abnormal reading is found, fear of hostility from patient or physician for straying outside a professional boundary and not seeing its relevance to the practice of dentistry. ${ }^{1}$ However, a majority of practitioners felt that education of dentists about hypertension was a good idea. About one third of practitioners who did not own equipment for measuring blood pressure still felt that such education was desirable. Hypertension or its treatment may produce adverse effects on various organ systems and management of the dental needs of these patients may require modification of routine treatment ${ }^{5}$ highlighting the importance of the condition to the dentist.

In the USA, professional boundaries can be more blurred. In one study of USA community dentists ${ }^{6} 26 \%$ of practitioners measured blood pressure on all new patients. An American GDP survey ${ }^{1}$ showed 33.5\% of respondents carrying out routine blood pressure measurement. The American dental literature has held a longstanding debate about screening for hypertension by dental practitioners. ${ }^{7,8}$ The consensus amongst USA practitioners (unlike in this survey) generally favours a move towards the screening of all patients, but the USA practitioners accept that there are potential pitfalls as well as benefits. One further potential problem referred to in the American literature ${ }^{7}$ (as well as those outlined above) is the criticism that the dental environment is unsuitable for measuring blood pressure since many patients find it threatening (thus increasing the likelihood of 'white coat hypertension'). Criticisms of 'white coat hypertension' have also been levelled at the general medical practice environment, however. It has been recognised for over 50 years that blood pressure measured in the patient's home is lower than that recorded by a doctor in the surgery. ${ }^{9}$ A review of previous studies has shown that false positive high blood pressure readings were no more common when taken in a dental surgery compared with other clinical settings. ${ }^{7}$ For a blood pressure to be diagnosed as truly elevated, the measurement should be confirmed on at least three occasions as the reliablity of measurements is improved if repeated measurements are made. ${ }^{10}$

Given that diagnosing and treating hypertension reduces morbidity and mortality, and that dentists could 'screen' their patients for hypertension, are the professional-cultural barriers too high for this to happen in the UK? Probably yes, since lines of professional demarcation may reflect differences in the UK and USA healthcare systems themselves. In the USA, a privately funded, privately provided system, it is 'every man for himself' and prevention is better (and cheaper) than cure. In the UK, there is less incentive to detect hidden disease (the state run system existing to some extent as a safety net).

So it would appear that blood pressure measuring equipment in many dentists' surgeries is more ornament than use. Rather than screening for hypertension, a more important consideration is that dentists consider measuring blood pressure in patients with a history of hypertension in order that safe patient management can be achieved.

Most GDPs in this study thought that education about hypertension was a good idea but did not want to be involved in any form of screening. This contrasts with the attitudes of USA dentists where opposition to regular blood pressure measurement in the dental environment is minimal.

The authors would like to thank Dr Nick Steen of the Centre for Health Services Research, University of Newcastle for his statistical advice and the practitioners who took the time to complete the questionnaires.

1. Ramaprasad R, Carson P H, Congdon E B, Barta PJ, Ziskin L Z. Dentists and blood pressure measurement: A survey of attitudes and practice. JAm Dent Assoc 1984; 108: 767-771.

2. Gortzak R A, Abraham-Inpijn L, ter Horst G, Peters G. High blood pressure screening in the dental office: A survey among Dutch dentists. Gen Dent 1993; 41: 246-251.

3. Brand HS, Abraham-Inpijn L. Cardiovascular responses induced by dental treatment. EurJ Oral Sci 1996; 104: 245-252.

4. Royal College of Surgeons of England. Guidelines for sedation by non-anaesthetists. London: The Royal College of Surgeons of England, 1993.

5. Cowper T R, Terezhalmy G T. Pharmacotherapy for hypertension. Dent Clin North Am 1996; 40: 585-610.

6. Pyle M A, Sawyer D R, Jasinevicius TR, Ballard R. Blood pressure measurement by Community Dentists. Spec Care Dent 1999; 19: 230-234.

7. Berman C L, Van Stewart A, Ramazotto L J, Davis F D. High blood pressure detection: A new Public Health measure for the dental profession. JAm DentAssoc 1976; 92: 116119 .

8. Secrest B G. Detecting, evaluating and treating hypertension. J Am Den Assoc 1994; 125: 104-106.

9. O'Brien E, Fitzgerald D. The history of blood pressure measurement. J Hum Hypertens 1994; 8: 73-84.

10. Beevers G, Lip G Y H, O'Brien E. ABC of hypertension: Blood pressure measurement. Part II - Conventional Sphygmomanometry: Technique of auscultatory blood pressure measurement. BrMed J 2001; 322: 1043-1047. 
1. How many years have you been in practice? years

2. Have you held/do you hold a hospital position?

I have held a hospital position in the past

I hold a hospital position now

Please tick

$\square \quad$ - Specialty

- Specialty...

3. Were you taught how to measure blood pressure
a) At Dental School?
b) As a postgraduate?
c) Both?
d) Not taught at all?

Please tick

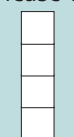

4. Do you specifically ask about blood pressure when taking a medical history?
Please tick

Yes

No

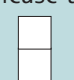

5. Does your surgery have equipment for measuring blood pressure?

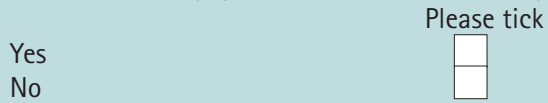

6. Do you routinely measure blood pressure on your patients?

Please tick

Yes

No

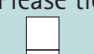

7. Do you measure blood pressure if there is a history of hypertension?

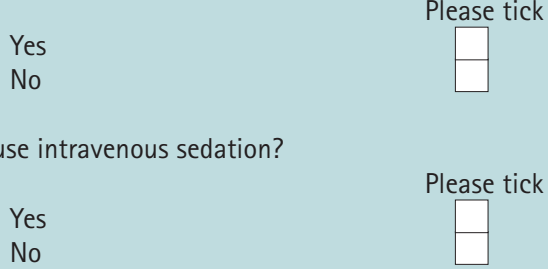

9. If yes, do you check a patient's blood pressure before administering sedation?

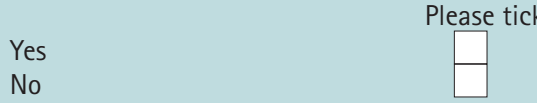

10. Do you think education of dental practitioners in the measurement of blood pressure is a good idea?

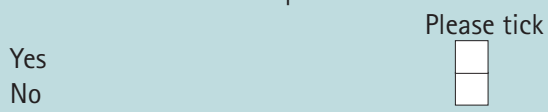

11. Do you think that screening of dental patients by dental practitioners for hypertension is a good idea?

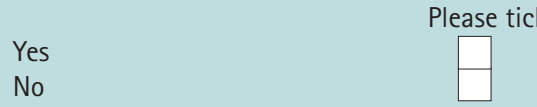

12. What do you consider to be a 'normal' blood pressure in a fit $70 \mathrm{Kg}$ adult. Please write freehand

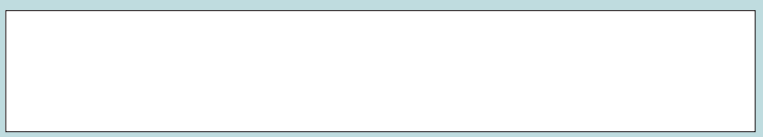

13. What medications are you aware of for the treatment of hypertension? Please state any known drugs by name, class or in any way you wish.

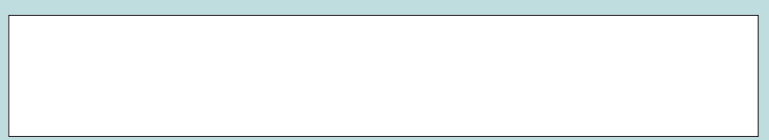

Thank you very much. Please return the questionnaire in the pre-paid envelope. 